\title{
Obstacle Avoidance Using Image Flow in an RT-Linux Environment on a PC-104 Platform
}

\author{
Jonathan R. Deming \\ Intelligent Systems and Robotics Group (ISRG) \\ New Mexico Institute of Mining and Technology \\ Socorro, NM 87801 \\ Email: nightowl@nmt.edu \\ Phone: (505) 835-6886
}

\author{
Stephen Bruder \\ Intelligent Systems and Robotics Group (ISRG) \\ New Mexico Institute of Mining and Technology \\ Socorro, NM 87801 \\ Email: bruder@nmt.edu \\ Phone: (505) 835-5272
}

\begin{abstract}
Semi-autonomous to fully autonomous robots rely on some form of data collection to operate in their environment. This has traditionally been accomplished using sonar or infra-red sensors to measure the robot's proximity to nearby objects. The most recent of efforts rely on sophisticated sensors, such as LIDAR and stereo vision, and result in solutions which are both expensive economically and computationally. Furthermore, these approaches often provide an overflow of data requiring a great deal of processing.

This paper discusses an alternative method using a single camera sampling images periodically to calculate the flow within an image and provide sufficient information to allow a small autonomous robot to navigate a corridor and react to obstacles. This method was implemented on a small robotic platform within an RT-Linux ${ }^{\mathrm{TM}}$ environment. Image data was collected using a CMU Cam.
\end{abstract}

Index Terms-Autonomous robots, image flow, image motion analysis, image processing, obstacle avoidance.

\section{INTRODUCTION}

$\mathrm{D}$ ESPITE recent advances in technology, robots are still very limited in their ability to sense obstacles in uncontrolled environments [1]. Sensing these objects is typically accomplished using sonar sensors which are immune to ambient light but exhibit limited directionality and accuracy [2]. Alternatively, stereoscopic vision can provide the data necessary with limited accuracy and at a very high processing cost [3], [4].

This paper discusses an approach to address this problem for the case where a robot is traveling down a corridor whose environmental input is provided by a single camera periodically sampling image data. The apparent flow within the image between frames is used to estimate relative motion. These motion cues can provide information about which corridor wall is closer to the robot in addition to whether or not something is immediately in front of the robot [6].

\footnotetext{
${ }^{\mathrm{TM}}$ WwW.fsmlabs.com

${ }^{\mathrm{T}}$ Www.diamondsystems.com
}

When the robot travels down a corridor the sides of the corridor will appear to diverge when looking in the direction of travel. This divergence is the greatest in the peripheral area of a forward looking image. The closer an object or corridor wall is to the camera, the faster it will move within the image. This motion is seen as image divergence or flow. By measuring the divergence within the peripheral areas of the image, a comparison can be made between the left and right sides of the image. The side with the greatest flow will represent the side of the corridor that is closest to the camera.

A similar approach can be used to determine when an object is getting closer to the camera. When approaching distant objects, they appear to get larger slower than when approaching them at a closer range. This rate of change can be measured using the central divergence of the image. When the central divergence exceeds some preset threshold, the robot should stop to avoid a collision. More robust optical flow based approaches have been suggested [5] wherein additional sensors can be used to supplement the monocular camera; however, inevitably a commensurate increase in complexity arises.

These methods were implemented in an RT-Linux environment on a PC-104 platform. Image data is provided in raw form by a CMU Camera through the serial port of a Diamond Systems ${ }^{\mathrm{TM}}$ Prometheus PC-104. The specifics of how this was done, issues that arose, and the results achieved will be discussed in the following sections.

\section{HARDWARE DESCRIPTION}

\section{A. Robotic Platform}

The robotic testing platform, shown in Figure 1, is a small cylindrical robot about $25 \mathrm{~cm}$ in diameter and $25 \mathrm{~cm}$ tall. Motion is provided by two DC motors independently driving two wheels configured for differential drive and stabilized by a third caster wheel. The robot is very maneuverable and is capable of spinning in place. Motor feedback is provided to the Prometheus via programmable logic that is interfaced with the PC-104 bus. The programmable logic also provides pulse width modulation to control the motors through an $\mathrm{H}$-Bridge. 


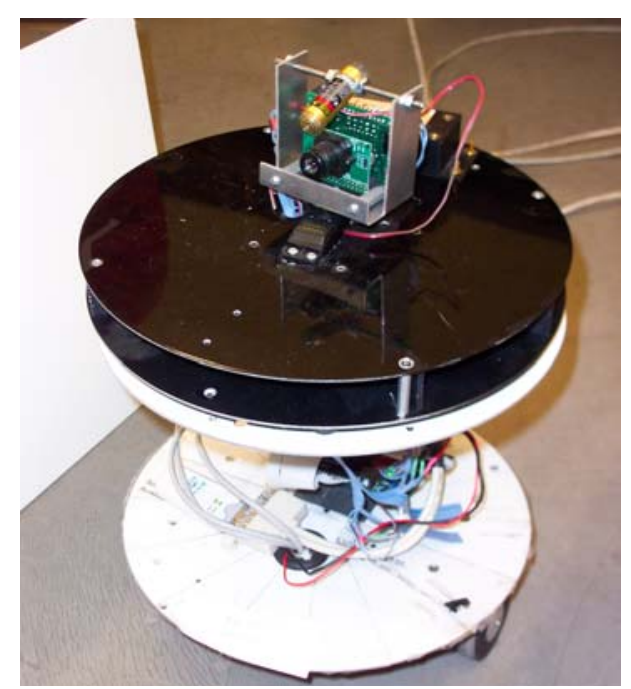

Figure 1: The robotic test platform.

\section{B. Computing Platform}

All image processing and system control is done on board the mobile robot using a Prometheus single board computer (SBC) running the RT-Linux operating system. The Prometheus SBC communicates with a stationary computer through a wireless USB based $802.11 \mathrm{~b}$ interface using SSH. All access to the software is made through this interface

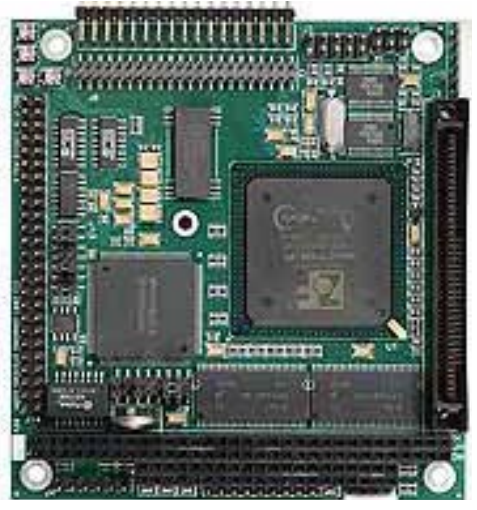
including the loading and execution of programs.

\section{CMU Camera}

Raw image data is collected using the CMU Camera developed by Carnegie Mellon University. The camera is designed to track objects but has additional features that allow it, among other things, to dump raw image data over the serial port. The camera samples at 17 frames per second at a resolution of $80 \times 143$. The field of view was measured experimentally to be about $40^{\circ}$ wide, with the standard lens. The raw image is sent serially column by column with a hex

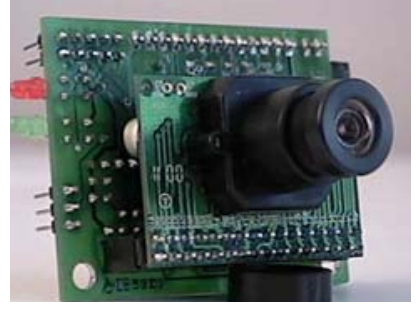
delimiter between columns. The raw image is $34.4 \mathrm{~Kb}$ and takes nearly 3 seconds to be transferred. This communications bottleneck results in data being sent from different frames. If a moving image is transmitted, smearing will occur. To solve this problem, a piecewise continuous motion is used and the robot is stopped each time an image is loaded. The camera is equipped with auto gain control which should be disabled before image data is loaded to optimize image quality.

\section{Testing Environment}

The image processing algorithm works best when there is a rich collection of unique features in the image with sufficiently fine detail. This is needed to ensure that each pixel cluster is traceable in the image and adequate large scale features exist so that at a long distance the limited resolution of the camera will be able to discern the features with enough depth for the algorithm to function properly. The CMU Camera has very limited resolution providing roughly only two pixels per degree of angular view. This limited resolution greatly reduces the amount of computation necessary, but, at the expense of performance. Figure 2 shows the view seen by the camera at the start of the corridor.

The corridor itself is constructed using brown cardboard with black marks and randomly torn pieces of paper and white Styrofoam blocks glued onto the sides of the corridor. The floor is gray featureless concrete. The area seen above the corridor walls is the lab in which the testing was conducted.

Other tested areas include a nearby hallway and the open lab floor. The hallway proved ineffective due to the lack of visual features between the periodic door jambs which provided a suitable reference. The polished floor combined with the bright fluorescent hallway lighting caused parts of the image to become saturated and effect the camera's performance. The lab environment would probably be more suitable if using a camera with higher resolution. Many of the features in the lab proved to be too small and blurry for them to be recognized by the image processing algorithm given the limited resolution.

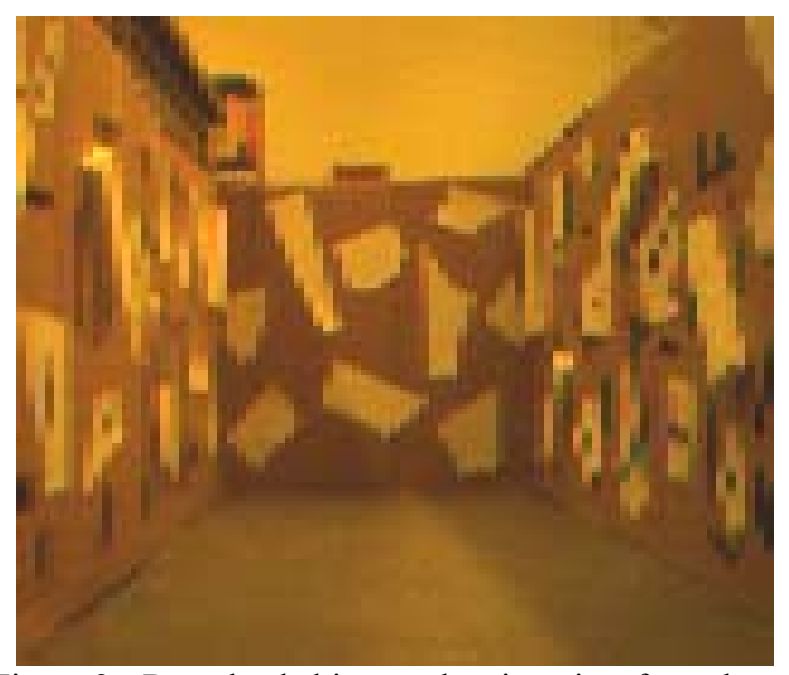

Figure 2: Downloaded image showing view from the start of the corridor as seen by the camera on the robot.

\section{AlgORITHM DESCRIPTION}

The following section will describe the algorithm used. Items not covered include trivial tasks such as the configuring of the serial port.

\section{A. Algorithm Overview}

The algorithm is first presented as a flow chart, in Figure 3, 
and then described textually in detail.

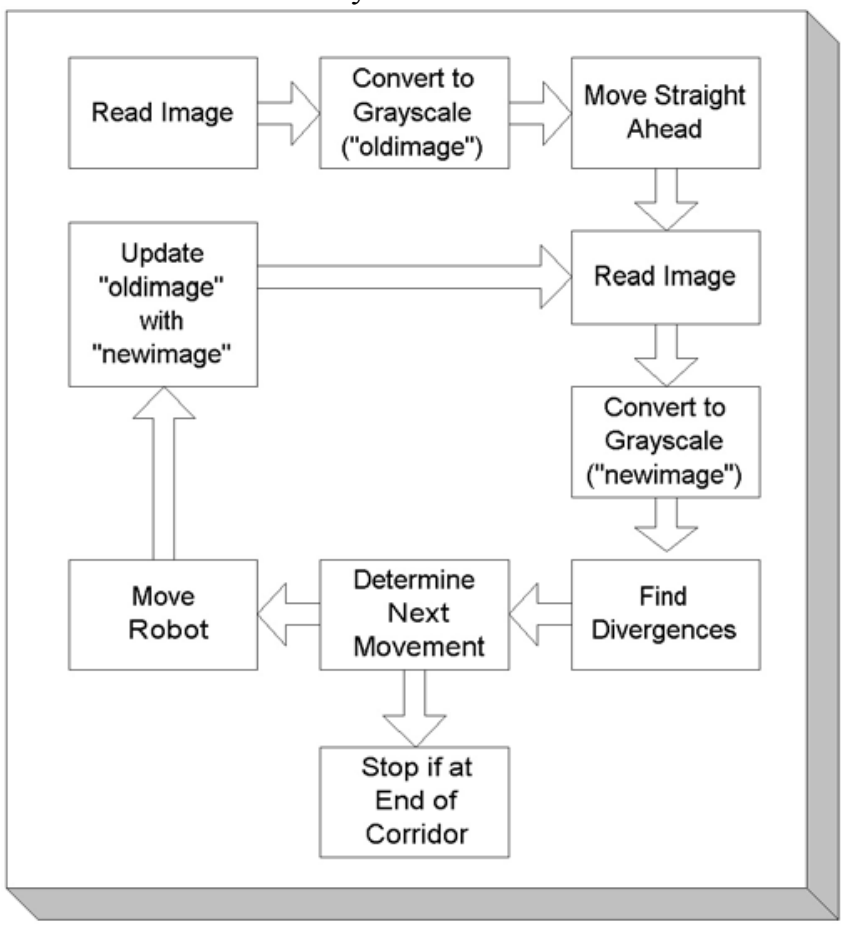

Figure 3: A flow chart of the main algorithm

The following sections will describe the non trivial steps in the algorithm in chronological order.

1. Send "DF" command to get new image.

2. Read image data.

3. Check for errors, if found, go to (1.).

4. Average red green and blue intensities of each pixel.

5. Convert serial data into a $80 \times 143$ array called "old-map".

6. Move robot straight ahead.

7. Send "DF" command to get new image.

8. Read image data.

9. Check for errors, if found, go to (7.).

10. Average red green and blue intensities of each pixel.

11. Convert serial data into a $80 \times 143$ array called "new-

12. Find the divergence within the image.

12.1. Break "new-image" into $10 \times 5$ pixel clusters

12.2. Find vertical and horizontal offset of each neighborhood

12.2.1. "Test fit" the cluster of "new-image" over its coordinate in "old-image" and every possible position \pm 5 pixels. Find deviation of each fit.

12.2.2. Find the best fit by choosing the fit with the minimum deviation. If the best fit has the same deviation as the position with zero shift, default to the zero shift position

13. Calculate left, right, and center divergence. Ignore offsets with greater than average deviation

13.1. Left Divergence $=-10 * \sum$ (horizontal shift of pixel clusters of 5 leftmost column in the first 12 rows whose deviation is less than average) / (\#of valid clusters).
13.2. Right Divergence $=10^{*} \sum$ (horizontal shift of pixel clusters of 5 rightmost column in the first 12 rows whose deviation is less than average) / (\#of valid clusters).

13.3. Central Divergence $=10 * \sum$ (vertical shift of lower 3 rows of pixel clusters.

14. Decide which way to go.
14.1. If ( Central Divergence $>0$ )
THEN: "End of Corridor" Stop Robot!

14.2. IF( Left Divergence $>$ Right Divergence +5 )

THEN: Turn Right

14.3. IF( Right Divergence $<$ Left Divergence +5 )

THEN: Turn Left

14.4. $\mathrm{IF}$ (Not turning or stopping)

THEN: Maintain Heading

15. Return to (1.).

\section{B. (2.) Read Image Data}

The CMU Cam transmits the raw image data by first sending and ASCII "ACK $\backslash \mathrm{r}$ " followed by hex data. The byte 0x01 indicates a new frame immediately followed by the redgreen-blue pixels of row one column one and then 142 more of these triples. Each column of data is sent sequentially separated by a hex-byte $0 \times 02$. The total stream including the "ACK $\backslash \mathrm{r}$ " is 34406 bytes long. In order to prevent early termination of the read process, the read function reads one byte at a time in a loop. The limitation of this approach is that when a byte is missed, say due to line noise, the read function is stuck in an infinite loop. To alleviate this problem, one less byte is read to allow for up to 1 byte to be lost without causing catastrophic problems. The program will still "hang" if more than one byte is dropped. The last character that gets dropped is a new frame indicator and can be ignored.

\section{C. (3.) Check for Errors}

Since there is no direct means of error-checking the transmitted data, say through the use of a checksum or similar technique, the best that can be done is to ensure that the column delimiters are in the location in which they are expected to be and each pixel is within the valid range of $0 \times 10$ to $0 \mathrm{xF} 0$. If these conditions are not met, an error flag is set and the image is resent before the robot is allowed to make another move.

The most common detectable error occurs when a byte is dropped resulting in a shift of all values in the string following this lost byte. If a byte is dropped in the first column sent, the entire image is corrupt while on the other hand if a pixel is dropped in the last column, only the remaining portion of the last column is corrupted.

\section{D. (4.) Average RGB Intensities of Each Pixel}

Once the downloaded data is considered valid, the red green and blue pixels are averaged together to determine the intensity of the given pixel. The CMU Camera has the option of sending chrominance data which is a weighted combination 
of the RGB values. The register in the camera that does this has been shown to be unreliable and since there is no reduction in the amount of data sent, the default setting was used to retrieve the separate RGB values and generate the composite intensity in the main program reliably.

For the purpose of debugging and having some user feedback a simple print function is used which sends either the HEX grayscale value of each pixel at the prompt or converts the grayscale image to a simple black and white array using a suitable threshold (set at about 75 or 80 ). The black and white text printout is much easier to visualize using 0 's and 1's. The black and white format also permits the use of plotting the change between images by using the exclusive-XOR of each pixel from "old-frame" and "new-frame". Note that these are for debugging purposes only and not used for the actual image processing.

\section{E. (5.) Convert Serial Data Into an 80x143 Array.}

This is a simple process using nested loops to map the serial grayscale data into a 2 dimensional array.

\section{F. (6.) Move Robot (straight ahead)}

Control software for the robot is rudimentary in nature. It is implemented in a function where arguments are passed to it which defines the peak power and distance to be traveled by each wheel. When the function is executed both wheels are linearly ramped up to their peak value and the distance traveled by each wheel is monitored in a loop that stops each motor once the distance traveled requirement is met. If the robot is to travel in a straight line, the power of each wheel is adjusted continuously to compensate for drift in either direction.

\section{G. (12.) Find the Divergence within the Image}

This step in the main algorithm is the principal component of the overall technique. It is broken into 2 major parts. The first part breaks "new-image" into 10x5 pixel clusters that are test fit against "old-image" in the second part to determine the horizontal and vertical offset of each cluster.

\section{1) (12.1) Break the Image Into 10x5 Pixel Clusters}

The "new-image" is broken up into small clusters $5 \times 10$ pixels in size. The first and last 5 rows and columns plus another 3 rows are cut away before splitting the image. This margin is to allow the test fitting process to determine if the clusters on the border of the image moved outwards into what would otherwise be out of frame. The additional 3 rows are left out to allow even symmetry in row breaks.

\section{2) (12.2) Find the Vertical and Horizontal Offset of Each} cluster.

This portion of the process is also broken into two separate parts. The first part test fits every possible position the cluster may move within a range of 5 pixels. Next, the best fit chosen based on the deviation calculated in the previous step.

\section{a) (12.2.1) Test Fit the Clusters}

Each cluster made from "new image" is separately test fit over 100 possible positions that cover up to a 5 pixel shift in each of the cardinal directions. The quality of fit is measured using a metric determined by the sum of the absolute difference between each pixel overlaid between the cluster and "old-map." This process is implemented using nested loops that provide a raster scan in which the position of +5 vertical offset and -5 horizontal offset is tested first.

\section{b) (12.2.2) Choose the Best Fit}

After each of the 100 possibilities has been tested, the fit with the smallest deviation is chosen. If this fit has equal deviation with the fit corresponding to no vertical or horizontal offset, the latter is chosen.

\section{H. (13.) Calculate Left, Right and Center Divergence}

Each of these three parameters is calculated independently but in a similar fashion.

1) (13.1) Left Divergence

The left divergence is found by summing the horizontal shift of the 5 leftmost and first 12 rows of clusters and multiplying by -10 over the number of valid clusters. Valid clusters are defined as those with less than average divergence.

2) (13.2) Right Divergence

The right divergence is found the same way at the left, however, the 5 rightmost columns of clusters are used instead and the value is multiplied by +10 .

3) (13.3) Central Divergence

The central divergence is calculated by summing the vertical shift of the lower 3 rows of pixel clusters in the image, and multiplying by 10 .

\section{I. (14) Decide Which Way to Go}

This part of the process is what gives the algorithm as a whole a means of output to the real world. The divergence values calculated in the previous section are used to determine whether to stop the robot, turn a fixed amount, or maintain heading for a fixed distance. The four options are outlined below.

\section{1) Stop}

If the central divergence as defined above is greater than zero, it indicates that the end of the corridor is close and the robot should stop.

\section{2) Turn Left}

If the left divergence is greater than the right divergence +5 , the robot is close to the right wall and should turn left.

\section{3) Turn Right}

If the right divergence is greater than the left divergence +5 , the robot is close to the right wall and should turn left.

\section{4) Maintain Heading}

If the robot has not stopped and the left and right divergences are within 5 of each other, the robot is a safe distance from both sides of the corridor and should maintain the current heading by driving straight ahead.

\section{J. Go to (1.)}

This simply closes the loop so that the robot will continue indefinitely until the end of the corridor is found. Once the 
end is found the test in (14.) will stop the robot and terminate the loop.

\section{RESUlTS}

This algorithm was tested extensively in the previously described corridor. Results show great promise although the robot did exhibit a less than perfect record. A variety of starting positions around the start of corridor were tried and tested along with varying starting orientations. It appears that whenever one side of the corridor is lost from the field of view of the camera, the divergence related to that wall becomes erroneous and tends to cause the robot to turn further in the wrong direction. The same behavior is seen when the robot gets to the end of the corridor without being centered in the corridor. Furthermore, whenever the robot came to close to the side of the corridor some of the features became too large and the pixel clusters became saturated with homogenous data resulting in a bad fit and the resulting flow was incorrect. Beyond these limitations, the greatest source of problems arose due to the corruption of data within the serial line. The XOR map showed any motion in an image independent of the divergence calculations. These maps show that the image contained noise significant enough to cause many of the grayscale values to cross the prescribed threshold value used in the binary map used for debugging. Significant effort was focused to minimize this problem; however, some residual effects still remained resulting in some tests working much better than others given the same starting conditions.

\section{CONCLUSION}

The test results indicate that this algorithm is valid and fast enough to allow for the extraction of the necessary information. Future work should include a camera with much higher resolution able to discriminate finer details in the environment. In order to work in a more unstructured environment the process described in (12.2.1) should be expanded beyond the small limit of \pm 5 pixels to a value that will exceed any "reasonable" image shift for the given application. A more involved process of picking the best fit should be developed to give preference to smaller divergence when the deviation of two fits is very close in an effort to minimize error as done in (12.2.2) where preference was given only to the zero-shift position when the divergences were equal.

In order to minimize the computing resources needed, the flow calculations could be limited to specific regions of interest in the field of view. Additionally, including optics to the camera to distort and stretch the edges of the image could be a possible way to visually amplify the divergence of the edge of the image.

\section{REFERENCES}

[1] D. Kortenkamp, R. P. Bonasso, and R. Murphey, "Artificial Intelligence and Mobile Robots: Case Studies of Sucessful Robot Systems," AAAI Press / The MIT Press, 1998.

[2] J. Borenstein, H. R. Everett, and , L. Feng, "'Where am I?' Sensors and Methods for Mobile Robot Positioning," Technical Report, The University of Michigan.

[3] Guilherme N. DeSouza and Avinash C. Kak, "Vision for Mobile Robot Navigation: A Survey," IEEE Trans. on Pattern Analysis and Machine Intelligence, Vol. 24, No. 2, Feb. 2002

[4] J.M. Saez and F. Escolano, "A global 3D map-building approach using stereo vision," Proceedings of the IEEE International Conference on Robotics and Automation, 2004.

[5] L. Sooyong and Jae-Bolc Song, "Robust mobile robot localization using optical flow sensors and encoders," Proceedings of the 2004 IEEE International Conference on Robotics and Automation, pp. 1039 - 1044, 26 April to May 1, 2004.

[6] C. McCarthy and N. Bames, "Performance of optical flow techniques for indoor navigation with a mobile robot," Proceedings of the 2004 IEEE International Conference on Robotics and Automation, pp. 5093- 5098, 26 April to May 1, 2004.

[7] D. Coombs, M. Herman, T. Hong, M. Nashrman. Real-Time Obstacle Avoidance Using Central Flow Divergence, and Peripheral Flow. IEEE Trans. on Robotics and Automation. Vol. 14. No. 1. February 1998.

[8] K. Weber, S. Venkatesh, M.V. Srinivasan. Insect Inspired Behaviours for the Autonomous Control of Mobile Robots. 